\title{
Correction: Genetic risk for schizophrenia and psychosis in Alzheimer disease
}

\author{
M. A. A. DeMichele-Sweet ${ }^{1}$ - E. A. Weamer ${ }^{2} \cdot$ L. Klei $^{1} \cdot$ D. T. Vrana ${ }^{3} \cdot$ D. J. Hollingshead ${ }^{4} \cdot$ H. J. Seltman ${ }^{5} \cdot$ R. Sims ${ }^{6}$.

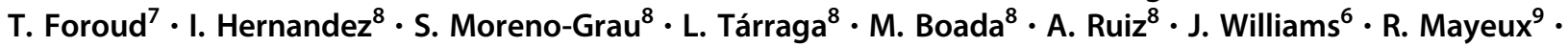 \\ O. L. Lopez ${ }^{1,2} \cdot$ E. L. Sibille ${ }^{1,10,11} \cdot$ M. I. Kamboh ${ }^{12} \cdot$ B. Devlin ${ }^{1} \cdot$ R. A. Sweet ${ }^{1,2,13}$
}

Published online: 12 March 2019

(c) Springer Nature Limited 2019

Correction to: Molecular Psychiatry (2018) 23, 963-972; https://doi.org/10.1038/mp.2017.81;

published online 2 May 2017

In the original version of this article, the following acknowledgements were accidentally omitted:

\section{"Genetic and Environmental Risk in AD Consortium 1 (UK):}

Data used in the preparation of this article were obtained from the Genetic and Environmental Risk for Alzheimer's Disease (GERAD1) Consortium [1]. The GERAD1 sample comprised up to $3941 \mathrm{AD}$ cases and 7848 controls. A subset of this sample has been used in this study. Cardiff University was supported by the Wellcome Trust, Medical Research Council (MRC) and/or Alzheimer's Research UK (ARUK) and the Welsh Assembly Government.

\section{GERAD1 contributors:}

Denise Harold ${ }^{1}$, Amy Gerrish ${ }^{1}$, Jade Chapman ${ }^{1}$, Valentina Escott-Price $^{1}$, Nandini Badarinarayan ${ }^{1}$, Richard Abraham ${ }^{1}$,

\section{R. A. Sweet}

sweetra@upmc.edu

1 Department of Psychiatry, University of Pittsburgh, Pittsburgh, PA, USA

2 Department of Neurology, University of Pittsburgh, Pittsburgh, PA, USA

3 Department of Computational Biology, Carnegie Mellon University, Pittsburgh, PA, USA

4 Genomics Research Core of the Health Sciences Core Research Facilities, University of Pittsburgh, Pittsburgh, PA, USA

5 Department of Statistics, Carnegie Mellon University, Pittsburgh, PA, USA

6 Division of Psychological Medicine and Clinical Neuroscience, School of Medicine, Cardiff University, Cardiff, UK
Paul Hollingworth ${ }^{1}$, Marian Hamshere ${ }^{1}$, Jaspreet Singh Pahwa $^{1}$, Kimberley Dowzell ${ }^{1}$, Amy Williams ${ }^{1}$, Nicola Jones $^{1}$, Charlene Thomas ${ }^{1}$, Alexandra Stretton ${ }^{1}$, Angharad Morgan $^{1}$, Kate Williams ${ }^{1}$, Sarah Taylor ${ }^{1}$, Simon Lovestone ${ }^{2}$, John Powell ${ }^{3}$, Petroula Proitsi ${ }^{3}$, Michelle K Lupton ${ }^{3}$, Carol Brayne $^{4}$, David C. Rubinsztein ${ }^{5}$, Michael Gill ${ }^{6}$, Brian Lawlor ${ }^{6}$, Aoibhinn Lynch $^{6}$, Kevin Morgan ${ }^{7}$, Kristelle Brown ${ }^{7}$, Peter Passmore ${ }^{8}$, David Craig ${ }^{8}$, Bernadette McGuinness ${ }^{8}$, Janet A. Johnston ${ }^{8}$, Stephen Todd ${ }^{8}$, Clive Holmes ${ }^{9}$, David Mann $^{10}$, A. David Smith ${ }^{11}$, Seth Love ${ }^{12}$, Patrick G. Kehoe ${ }^{12}$, John Hardy ${ }^{13}$, Rita Guerreiro ${ }^{14,15}$, Andrew Singleton ${ }^{14}$, Simon Mead ${ }^{16}$, Nick Fox ${ }^{17}$, Martin Rossor ${ }^{17}$, John Collinge ${ }^{16}$, Wolfgang Maier ${ }^{18}$, Frank Jessen ${ }^{18}$, Reiner Heun ${ }^{18}$, Britta Schürmann ${ }^{18,19}$, Alfredo Ramirez ${ }^{18}$, Tim Becker ${ }^{20}$, Christine $\operatorname{Herold}^{20}$, André Lacour ${ }^{20}$, Dmitriy Drichel ${ }^{20}$, Hendrik van den Bussche ${ }^{21}$, Isabella Heuser ${ }^{22}$, Johannes Kornhuber $^{23}$, Jens Wiltfang ${ }^{24}$, Martin Dichgans ${ }^{25,26}$, Lutz Frölich $^{27}$, Harald Hampel ${ }^{28,29}$, Michael Hüll ${ }^{30}$, Dan Rujescu $^{31}$, Alison Goate ${ }^{32}$, John S.K. Kauwe ${ }^{33}$, Carlos Cruchaga $^{34}$, Petra Nowotny ${ }^{34}$, John C. Morris ${ }^{34}$, Kevin Mayo $^{34}$, Gill Livingston ${ }^{35}$, Nicholas J. Bass ${ }^{35}$, Hugh

7 Medical and Molecular Genetics, Indiana University School of Medicine, Indianapolis, IN, USA

8 Research Center and Memory Clinic of Fundació ACE, Institut Català de Neurociències Aplicades, Barcelona, Spain

9 Departments of Neurology, Psychiatry and Epidemiology, Columbia University, New York, NY, USA

10 Department of Psychiatry and Pharmacology and Toxicology, University of Toronto, Toronto, ON, Canada

11 Campbell Family Mental Health Research Institute of CAMH, Toronto, ON, Canada

12 Department of Human Genetics, University of Pittsburgh, Pittsburgh, PA, USA

13 VISN 4 Mental Illness Research, Education and Clinical Center (MIRECC), VA Pittsburgh Healthcare System, Pittsburgh, PA, USA 
Gurling ${ }^{35}$, Andrew McQuillin ${ }^{35}$, Rhian Gwilliam ${ }^{36}$, Panagiotis Deloukas ${ }^{36}$, Markus M. Nöthen ${ }^{20}$, Peter Holmans ${ }^{1}$, Michael O'Donovan ${ }^{1}$, Michael J. Owen ${ }^{1}$

${ }^{1}$ Medical Research Council (MRC) Centre for Neuropsychiatric Genetics and Genomics, Neurosciences and Mental Health Research Institute, Department of Psychological Medicine and Neurology, School of Medicine, Cardiff University, Cardiff, UK. ${ }^{2}$ Department of Psychiatry, Medical Sciences Division, University of Oxford, Oxford, UK. ${ }^{3}$ Kings College London, Institute of Psychiatry, Department of Neuroscience, De Crespigny Park, Denmark Hill, London, UK. ${ }^{4}$ Institute of Public Health, University of Cambridge, Cambridge, UK. ${ }^{5}$ Cambridge Institute for Medical Research, University of Cambridge, Cambridge, UK. ${ }^{6}$ Mercers Institute for Research on Aging, St. James Hospital and Trinity College, Dublin, Ireland. ${ }^{7}$ Institute of Genetics, Queens Medical Centre, University of Nottingham, UK. ${ }^{8}$ Ageing Group, Centre for Public Health, School of Medicine, Dentistry and Biomedical Sciences, Queens University Belfast, UK. ${ }^{9}$ Division of Clinical Neurosciences, School of Medicine, University of Southampton, Southampton, UK. ${ }^{10}$ Clinical Neuroscience Research Group, Greater Manchester Neurosciences Centre, University of Manchester, Salford, UK. ${ }^{11}$ Oxford Project to Investigate Memory and Ageing (OPTIMA), University of Oxford, Department of Pharmacology, Mansfield Road, Oxford, UK. ${ }^{12}$ University of Bristol Institute of Clinical Neurosciences, School of Clinical Sciences, Frenchay Hospital, Bristol, UK. ${ }^{13}$ Department of Molecular Neuroscience and Reta Lilla Weston Laboratories, Institute of Neurology, UCL, London, UK. ${ }^{14}$ Laboratory of Neurogenetics, National Institute on Aging, National Institutes of Health, Bethesda, Maryland, United States of America. ${ }^{15}$ Department of Molecular Neuroscience, Institute of Neurology, University College London, Queen Square, London WC1N 3BG, UK. ${ }^{16}$ MRC Prion Unit, Department of Neurodegenerative Disease, UCL Institute of Neurology, London, UK. ${ }^{17}$ Dementia Research Centre, Department of Neurodegenerative Diseases, University College London, Institute of Neurology, London, UK. ${ }^{18}$ Department of Psychiatry, University of Bonn, Sigmund-Freud-Straße 25, 53105 Bonn, Germany. ${ }^{19}$ Institute for Molecular Psychiatry, University of Bonn, Bonn, Germany. ${ }^{20}$ Department of Genomics, Life \& Brain Center, University of Bonn, Bonn, Germany. ${ }^{21}$ Institute of Primary Medical Care, University Medical Center Hamburg-Eppendorf, Germany. ${ }^{22}$ Department of Psychiatry, Charité Berlin, Germany. ${ }^{23}$ Department of Psychiatry, Friedrich-Alexander-University Erlangen-Nürnberg, Germany. ${ }^{24}$ Department of Psychiatry and Psychotherapy, University Medical Center (UMG), Georg-August-University, Göttingen, Germany. ${ }^{25}$ Institute for Stroke and Dementia Research, Klinikum der Universität München, Marchioninistr. 15, 81377, Munich, Germany.
${ }^{26}$ Department of Neurology, Klinikum der Universität München, Marchioninistr. 15, 81377, Munich, Germany. ${ }^{27}$ Central Institute of Mental Health, Medical Faculty Mannheim, University of Heidelberg, Germany. ${ }^{28}$ Institute for Memory and Alzheimer's Disease \& INSERM, Sorbonne Universities, Pierre and Marie Curie University, Paris, France. ${ }^{29}$ Institute for Brain and Spinal Cord Disorders (ICM), Department of Neurology, Hospital of Pitié-Salpétrière, Paris, France. ${ }^{30}$ Centre for Geriatric Medicine and Section of Gerontopsychiatry and Neuropsychology, Medical School, University of Freiburg, Germany. ${ }^{31}$ Department of Psychiatry, University of Halle, Halle, Germany. ${ }^{32}$ Neuroscience Department, Icahn School of Medicine at Mount Sinai, New York, USA. ${ }^{33}$ Department of Biology, Brigham Young University, Provo, UT, 84602, USA. ${ }^{34}$ Departments of Psychiatry, Neurology and Genetics, Washington University School of Medicine, St Louis, MO 63110, USA. ${ }^{35}$ Department of Mental Health Sciences, University College London, UK. ${ }^{36}$ The Wellcome Trust Sanger Institute, Wellcome Trust Genome Campus, Hinxton, Cambridge, UK

\section{Fundació ACE Barcelona Alzheimer Treatment and Research Center (ACE):}

We thank all patients for their participation in this project. We are obliged to Trinitat Port-Carbó and her family for their support of the Fundació ACE research programs. Fundació ACE collaborates with the Centro de Investigación Biomédica en Red sobre Enfermedades Neurodegenerativas (CIBERNED, Spain), and is one of the participating centers of the Dementia Genetics Spanish Consortium (DEGESCO). CIBERNED is an Instituto de Salud Carlos III ISCIII Project. AR is supported by Grant PI13/02434 (Acción Estratégica en Salud, Instituto de Salud Carlos III (ISCIII), Ministerio de Economía y Competitividad, Spain) and Fundación Bancaria "La Caixa" (Barcelona, Spain).

Fundació ACE Contributors (non authors): Asunción Lafuente, Octavio Rodríguez-Gómez, Liliana Vargas, Carla Abdelnour, Domingo Sánchez, Ana Mauleón, Maitée Rosende-Roca, Silvia Gil, Montserrat Alegret, Ana Espinosa, Gemma Ortega, Ángela Sanabria, Alba Pérez, Marta Ibarria, Susana Diego, María José de Dios, Pilar Canyabate, Mariola Moreno, Sílvia Preckler, Oscar Sotolongo-Grau, Itziar de Rojas, Elvira Martín, Susana Ruiz, Marina Tarragona, Judit Serra.

\section{National Institute of Mental Health Genetics Initiative AD Cohort (NIMH):}

Data and biomaterials were collected in three projects that participated in the National Institute of Mental Health (NIMH) Alzheimer Disease Genetics Initiative. From 1991 to 1998, the Principal Investigators and Co-Investigators were: Massachusetts General Hospital, Boston, MA, U01 
MH46281, Marilyn S. Albert and Deborah Blacker; Johns Hopkins University, Baltimore, MD; U01 MH46290, Susan S. Bassett, Gary A. Chase, and Marshal F. Folstein; University of Alabama, Birmingham, AL, U01 MH46373, Rodney C.P. Go and Lindy E. Harrell.

\section{Cardiovascular Health Study (CHS):}

This research was supported by contracts HHSN268201200036C, HHSN268200800007C, N01HC55222, N01HC85079, N01HC85080, N01HC85081, N01HC85082, N01HC85083, N01HC85086, and grants U01HL080295 and U01HL130114 from the National Heart, Lung, and Blood Institute (NHLBI), with additional contribution from the National Institute of Neurological Disorders and Stroke (NINDS). Additional support was provided by R01AG023629 from the National Institute on Aging (NIA). A full list of principal CHS investigators and institutions can be found at CHS-NHLBI.org. This manuscript has been reviewed by CHS for scientific content and consistency of data interpretation with previous CHS publications.

National Institute on Aging's Late Onset Alzheimer's Disease Family Study (NIA-LOAD) and a consortium of National Institute on Aging Alzheimer Disease Centers (ADC), including the National Alzheimer's Coordinating Center, The National Cell Repository for Alzheimer's Disease, and the Alzheimer's Disease Genetics Consortium:

The NACC database is funded by NIA/NIH Grant U01 AG016976. NACC data are contributed by the NIA-funded ADCs: P30 AG019610 (PI Eric Reiman), P30 AG013846 (PI Neil Kowall), P50 AG008702 (PI Scott Small), P50 AG025688 (PI Allan Levey), P50 AG047266 (PI Todd Golde), P30 AG010133 (PI Andrew Saykin), P50 AG005146 (PI Marilyn Albert), P50 AG005134 (PI Bradley Hyman), P50 AG016574 (PI Ronald Petersen), P50 AG005138 (PI Mary Sano), P30 AG008051 (PI Steven Ferris), P30 AG013854 (PI M. Marsel Mesulam), P30 AG008017 (PI Jeffrey Kaye), P30 AG010161 (PI David Bennett), P50 AG047366 (PI Victor Henderson),
P30 AG010129 (PI Charles DeCarli), P50 AG016573 (PI Frank LaFerla), P50 AG016570 (PI Marie-Francoise Chesselet), P50 AG005131 (PI Douglas Galasko), P50 AG023501 (PI Bruce Miller), P30 AG035982 (PI Russell Swerdlow), P30 AG028383 (PI Linda Van Eldik), P30 AG010124 (PI John Trojanowski), P50 AG005133 (PI Oscar Lopez), P50 AG005142 (PI Helena Chui), P30 AG012300 (PI Roger Rosenberg), P50 AG005136 (PI Thomas Montine), P50 AG033514 (PI Sanjay Asthana), P50 AG005681 (PI John Morris), and P50 AG047270 (PI Stephen Strittmatter).

Samples from the National Cell Repository for Alzheimer's Disease (NCRAD), which receives government support under a cooperative agreement grant (U24 AG21886) awarded by the National Institute on Aging (NIA), were used in this study. We thank contributors who collected samples used in this study, as well as patients and their families, whose help and participation made this work possible.

The Alzheimer's Disease Genetics Consortium supported the collection of samples used in this study through National Institute on Aging (NIA) grants U01AG032984 and RC2AG036528.

The NIA-LOAD study supported the collection of samples used in this study through National Institute on Aging (NIA) grants U24AG026395 and R01AG041797. We thank contributors, including the Alzheimer's Disease Centers who collected samples used in this study, as well as patients and their families, whose help and participation made this work possible."

The authors would like to apologise for this error. This has not been corrected in the PDF or HTML of this article.

\section{References}

1. Harold D, Abraham R, Hollingworth P, Sims R, Gerrish A, Hamshere ML, et al. Genome-wide association study identifies variants at CLU and PICALM associated with Alzheimer's disease. Nat Genet. 2009;41:1088-93. 